Pbar Note \#627

\title{
Design of the 0.5 - 1 GHz Planar Recycler Pickup and Kicker Antennas
}

\author{
C. Deibele
}

\begin{abstract}
The stochastic cooling system in the Recycler ring at Fermilab required the addition of a $0.5-1 \mathrm{GHz}$ cooling system. This requirement dictated the design of a new antenna for this band of the system. The design problem is defined, method of design is illustrated, and the measurement data are reported.
\end{abstract}

\section{Introduction}

The Recycler is a storage ring comprised of mostly permanent magnets located in the tunnel of the Main Injector at Fermilab. The goal for the construction of the Recycler is to collect and store unused antiprotons from collisions in the Tevatron for use in future collisions in the Tevatron. It will both stochastically and electron cool these unused antiprotons before another collision experiment is possible in the Tevatron. By reusing the antiprotons the luminosity of the experiment can be increased faster.

The Recycler will use three bands for its stochastic cooling system. It will reuse the existing designs from the Antiproton Source for the 1-2 GHz and 2-4 GHz systems ${ }^{1}$, and it requires a new design for an additional lower frequency band for the $0.5-1 \mathrm{GHz}$ system. Since the existing designs were fabricated using a microstrip topology it was desired that the new design use a similar topology so that the vacuum tank designs and supporting hardware be identical for all three bands.

A primary difference between the design of the pickups/kickers of the Antiproton Source and the Recycler is a different aperture in the machine itself. The Recycler has a bigger aperture and

${ }^{1}$ D. P. McGinnis, Theory and Design of Microwave Planar Electrodes for Stochastic Cooling of Particle Beams, Microwave and Optical Technology Letters, Vol. 4, No. 11, October 1991. 
consequently reusing the designs for the existing Antiproton Source pickups/kickers is not electrically optimal but is cost efficient. Measurements will be shown later in this paper for the design of the $0.5-1$ $\mathrm{GHz}$ system showing the effect of the aperture on the antenna performance.

A mockup of the Recycler tank was manufactured for designing and testing the $0.5-1 \mathrm{GHz}$ pickups/kickers. The design procedure was an iterative process and required both a constant dialogue and also a strong relationship with a boart house for the manufacture of each iterative step. It also required extensive use of time domain reflectometers (TDRs) and vector network analyzers (NWAs). The TDR was used for impedance matching and for a rough measurement of the center frequency of the antenna. The testing process also used the theory of stretched wire measurements ${ }^{2}$ (SWM) for finding th exact center frequency of the pickups/kickers as well as the beam coupling

to the antenna.

Figure 1. A picture of a current $1-2 \mathrm{GHz}$ pickup/kicker antenna.

\section{Design Theory of Kickers/Pickups}

The blue areas are copper and are located a. Background on the beam side of the beampipe. The red

When designing any structure for any application, it is first requiredareas are located on the microstrip side of to first know how big the design can be and also what the cost requirements the board and face away from the beam. that the design can have. For example, in the Recycler it was desired that the antenna be no larger than the horizontal/vertical aperture of the beam. The black areas are dielectric. The beam travels across this antenna from left to right.

${ }^{2}$ Sands, Rees, SLAC-report PEP-95, August 1974. 
The largest aperture was predicted to be circa $50 \mathrm{~mm}$ and therefore the antenna has constraint to be no larger than $50 \mathrm{~mm}$. For background topological information of what a pickup/kicker looks like, a picture of an Antiproton Source 1-2 GHz antenna is shown in Fig. 1. It is also desired for the antenna to have an impedance as large as possible so that the coupled signal from the beam is as large as possible. Having a high $\mathrm{R}_{\text {antenna }}$ is related to a signal/noise constraint and is determined via the relationship:

$$
\mathrm{P}_{\text {couple }}=\mathrm{I}_{\text {beam }}^{2} \mathrm{R}_{\text {antenna }} \text {. }
$$

Since the $\mathrm{I}_{\text {beam }}$ term is a constant, then the largest coupled power would have the highest antenna impedance $R_{\text {antenna }}$. Since the Antiproton Source pickups/kickers were chosen to be $100 \Omega$, it was decided that the new Recycler design should have the same impedance. Because standard measurement equipment has a $50 \Omega$ impedance it was required to design a matching network to match from the standard measurement devices to the antenna impedance and then back to a $50 \Omega$ load.

The operation of a pickup/kicker is easily described with the theory of coupled lines rather than with standard antenna theory. The time domain response of a pickup/kicker is

$$
\frac{\mathrm{C}_{\text {coup }}}{2}\left(\delta(\mathrm{t})-\delta\left(\mathrm{t}-\frac{2 \mathrm{~L}}{\mathrm{c}}\right)\right) .
$$

The term $\mathrm{C}_{\text {coup }}$ is the coupling from the beam to the pickup/kicker, $\mathrm{L}$ is the length of the pickup/kicker, and $\mathrm{c}$ is the speed of light. $\mathrm{C}_{\text {coup }}$ is a constant and is strongly dependant on the geometrical size of the pickup/kicker.

\section{b. TDR Measurements}

Because the present designs of pickups/kickers for the Antiproton Source are relatively weakly coupled to the beam (on the order of -15 to $-22 \mathrm{~dB}$ ), the odd and even mode impedances of the 
coupling antenna are similar. ${ }^{3}$ The implication of weak coupling makes the implementation and matching of the coupler easier. The matching can be done straightforwardly by operating the antenna in its different modes, namely with a TDR for impedance matching and with a NWA for a through measurement for determining the antenna center frequency.

The TDR is a tool used to ensure that the antenna is well matched. A schematic of a TDR setup is shown in Fig. 2. Because the impedance of the antenna is desired to be as large as possible, matching networks are required to match the antenna to standard measurement equipment. The chosen matching network depends on the center frequency of the antenna. For a 4-8 GHz band, the wavelength is short enough such that for practical purposes, a linear microstrip taper from $50 \Omega$ to $100 \Omega$ is a sufficient matching network. For the $0.5-1 \mathrm{GHz}$ antenna it was required to design a three $\lambda 4$ stage Chebyshev matching network. A similar network is also required on the output of the

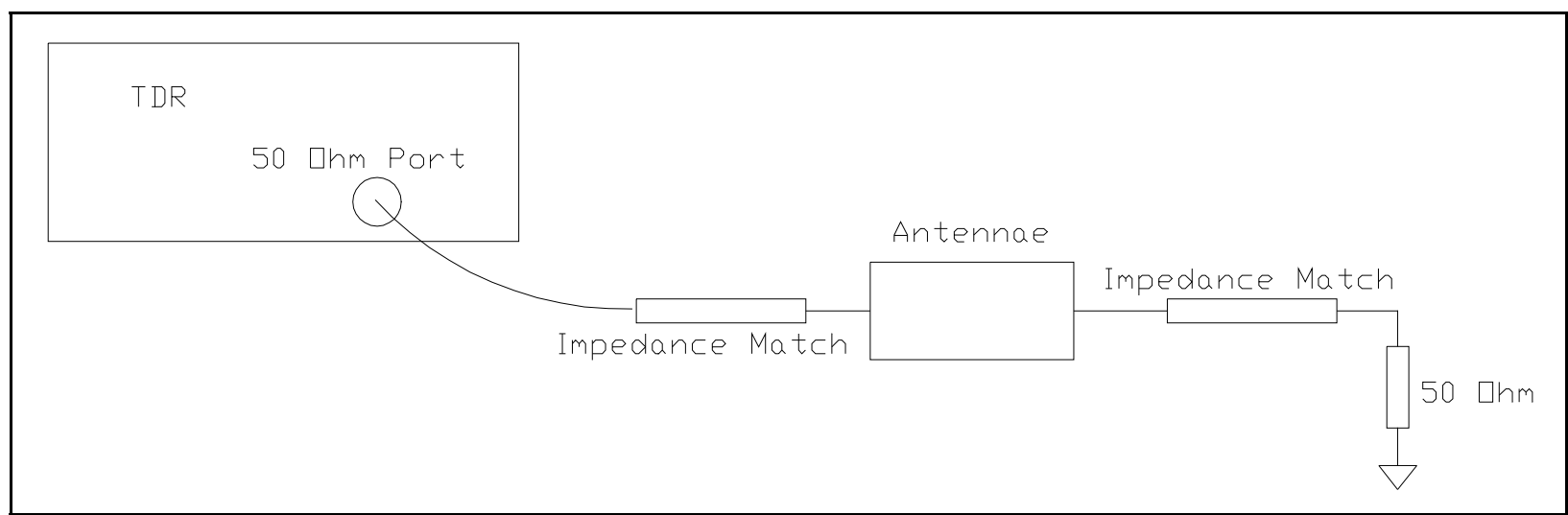

Figure 2. A schematic of the setup for testing the impedance of the antennae. The realization of the impedance matching networks are dependant on the bandwidth and center frequency of the antennae. A high center frequency antennae (i.e. $6 \mathrm{GHz}$ ) can be formed with simple linear tapers. Lower center frequency antennae may require $\lambda / 4$ wave Chebyschev matching networks.

${ }^{3}$ Brown, Sharpe, Hughes, Post, "Lines, Waves, and Antennas," John Wiley and Sons, Second Edition, pp. 136-145. 


\begin{abstract}
antenna to match
from the $100 \Omega$

antenna to the $50 \Omega$

load. The

impedance matching

networks on each
\end{abstract}

side of the antenna

for the Antiproton

Source are mirror

images of each other.

The electrical length of the antenna may be crudely measured directly from a TDR steps from the Chebyshev matching networks, and the antenna is the portion of the graph where the impedance is $100 \Omega$. The center frequency can be calculated from the time difference from the last part of the first matching network to the first part of the last matching network. This is to say that the ideal antenna in Fig. 3 begins at $\mathrm{t}=2.5 \mathrm{nsec}$ and ends at $\mathrm{t}=3.5 \mathrm{nsec}$.

Determining the total electrical length of the antenna of Fig. 3 requires careful consideration for the measurement technique. A TDR, by definition, measures a reflected signal based on the time difference from the time the signal is transmitted from the TDR to the time the signal returns back at the sample head on the TDR. Therefore, the time-length of a structure measured by a TDR is actually two times less than the measured length. This means that the time-length of the antenna in Fig. 3 is 


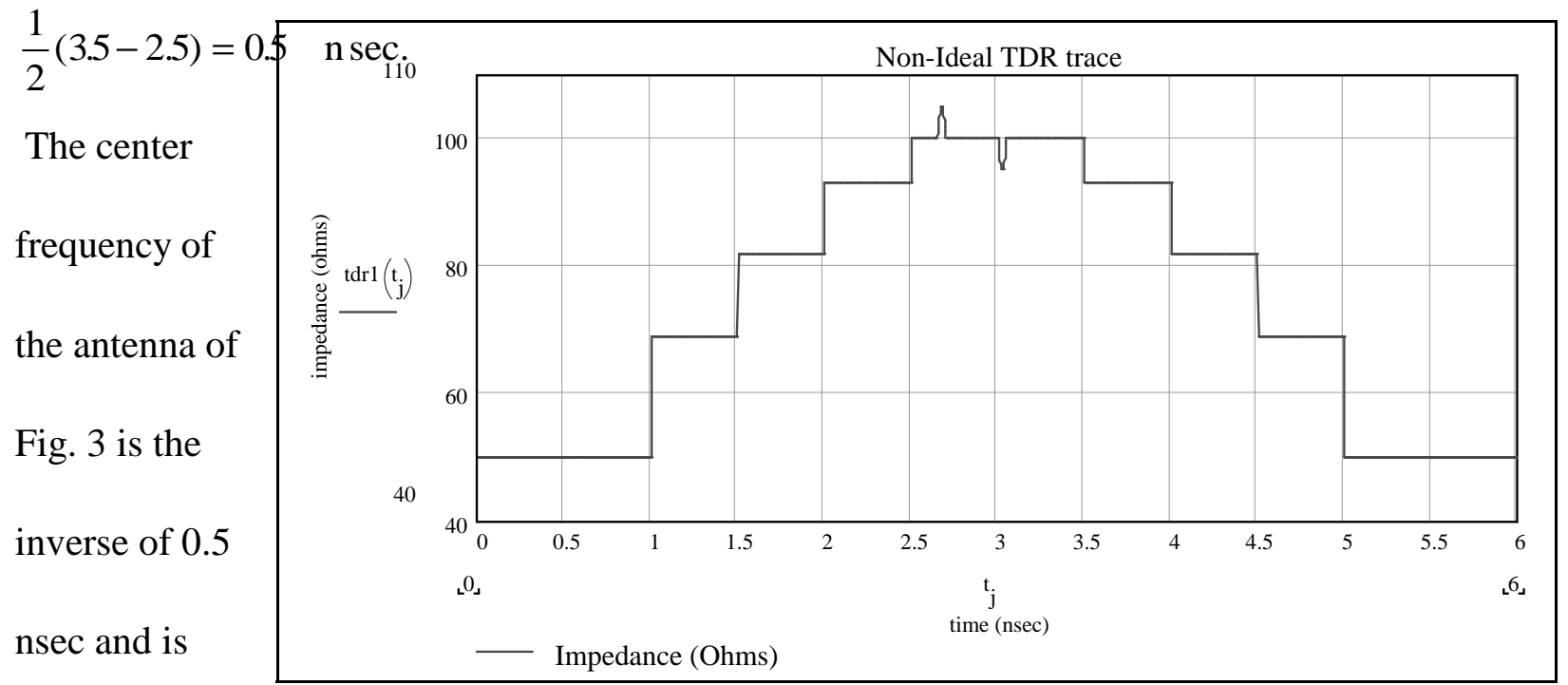

therefore 2

Figure 4. A non-ideal antenna with matching networks. The small blips at $\mathrm{t}=2.65$ nsec and $t=3$ nsec show its non-ideal behavior. The positive blip at $t=2.65$ is

GHz. The

electrical inductive and represents a lack of copper. Correction of this blip requires a small amount of copper tape on the antenna. The negative blip at $t=3 \mathrm{nsec}$ is capacitive. Correction of capacitive blips requires removal of copper on the antenna.

length from Eq. 1 of the antenna is calculated by

$$
\text { electrical length }=\mathrm{c}_{\text {light }} \text { time } .
$$

So far only an ideal antenna has been considered. A contrived example of a non-ideal antenna is shown in Fig. 4. The salient features of the graph of Fig. 4 is a small positive blip at $t=2.65 \mathrm{nsec}$ and a negative blip at $\mathrm{t}=3 \mathrm{nsec}$. The positive blip, or inductive discontinuity, is a local phenomenon and can be removed the TDR plot by adding a small piece of conductor at a distinct position on the antenna. This position can be experimentally determined by moving a sharp conductive point (like a razor blade or dentist scraper) along the antenna. Placement of a conductor on the antenna will introduce a sharp discontinuity on the TDR plot. Sliding the conducting point along the antenna moves the conductingpoint-induced-discontinuity along the TDR plot. When the additional discontinuity lies at the same time position where the positive blip exists then the position of the mismatch is found. At this mismatch point 
a small piece of

conductor must be

added to the

inductive mismatch

to produce a better

match. The negative

blip, or capacitive

mismatch, at $\mathrm{t}=3$

nsec is also a local

phenomenon and

can be removed by

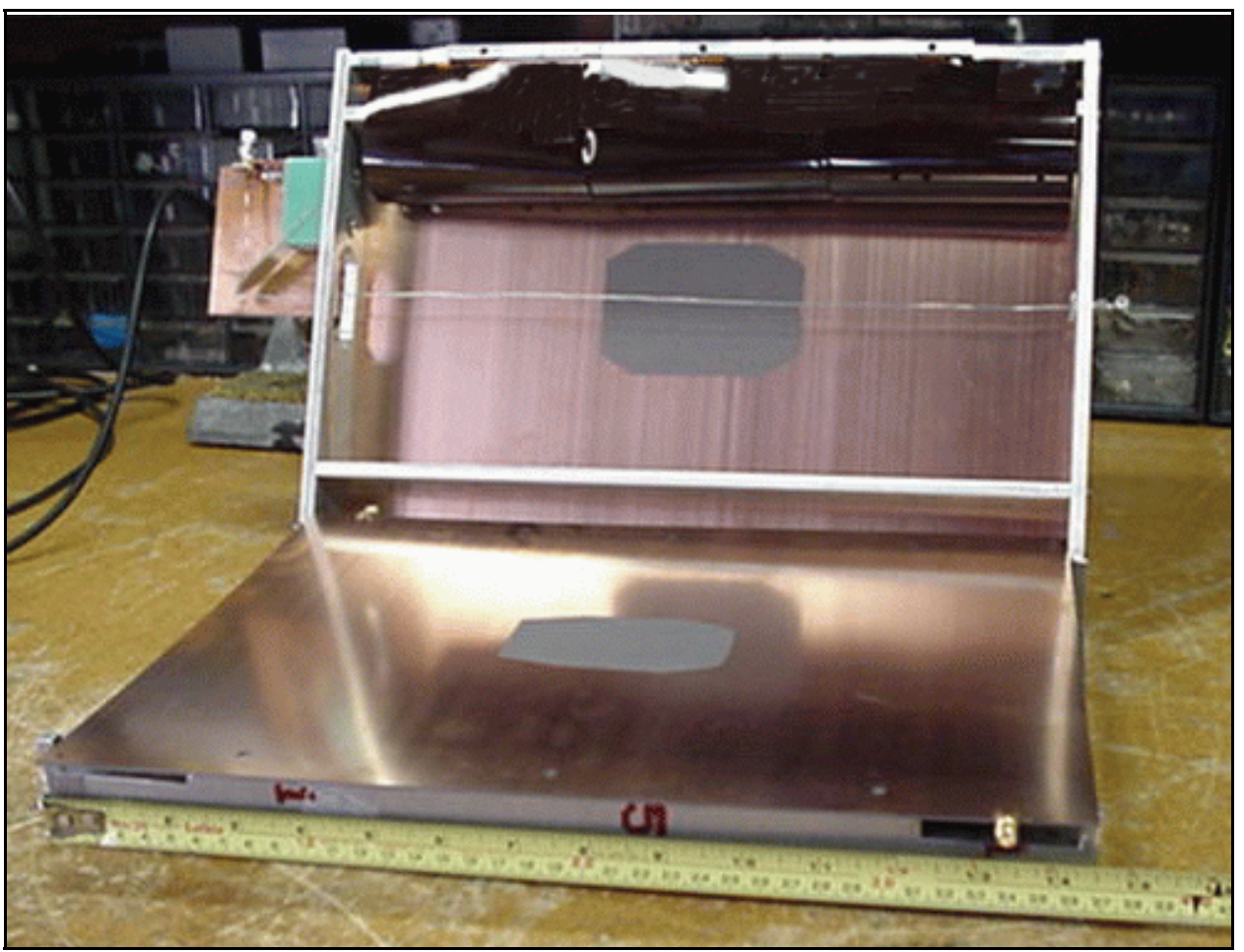

Figure 5. The assembly for simulating the response of the antenna to the beam by using a stretched wire measurement. At the top of the photo are some resistively coated kapton pieces to absorb higher order modes caused by the

removing a small mismatches. Note that the assembly is taken apart and usually the two pieces are held together for measurement purposes.

piece of conductor

at a distinct point. The procedure for determining the location of the capacitive mismatch follows

exactly as for the inductive blip.

\section{c. NWA Measurements}

Using the theory outlined by Sands and Rees, it is desired to simulate the response of the antenna using a stretched wire measurement (SWM). A picture of the setup used for the 0.5-1 GHz setup is shown in Fig. 5. The top part of the assembly was removed to show the wire and the resistively coated kapton pieces. The resistors were added to absorb higher order modes caused by mismatches of the wire with the assembly and any waveguide modes which may be launched into the 
measurement area. On the top piece of the assembly at the left hand side is a small setup to hold the wire taut for each measurement. With the setup of Fig. 5, a signal is sent into the right hand connector and onto the center wire. The output signal is measured on the front right hand connector on the circuit board.

A problem intrinsic to using the SWM technique involves de-embedding the measurement. Using an NWA to measure the response of the antenna involves several assumptions. First, the measurement has a large discontinuity (from a $50 \Omega$ coaxial line to the wire in the assembly which is circa $200 \Omega$. This discontinuity cause rather large reflection and cannot be avoided using the SWM technique unless a matching network is used. Second, the output si of the wire suffers from a discontinuity and causes reflections. The reflections will travel back over the antenna and can cause a misinterpretation of the measured results. This effect can be minim by using a long test assembly and using gating techniques on the NWA. Finally, the matching network of Fig. 2 causes multiple reflections, and is band limited.

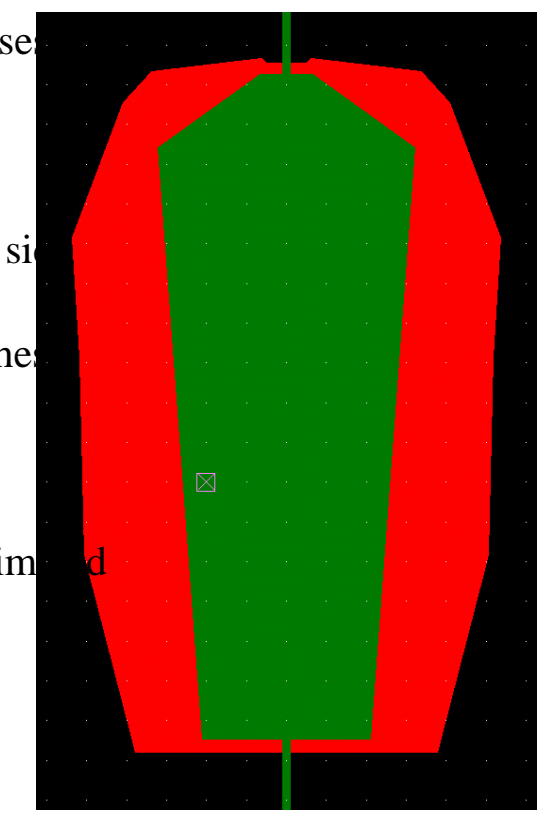

Figure 6. The $0.5-1 \mathrm{GHz}$ antenna. The green areas are copper and are on the

To overcome all of these shortcomings with the SWM, it is microstrip side of the antenna. The red area is dielectric and necessary to analyze the SWM data in the time domain and to gate out black area is copper and is the reflected erroneous signals. This technique also requires that a located on the beam side of the pickup. The beam would travel from the top of the broad-band measurement is made so that proper resolution can be picture to the bottom. This is made to gate out the correct signals. Note that this doesn't quite give picture in Fig. 6. the same antenna shown in the 
the complete answer but

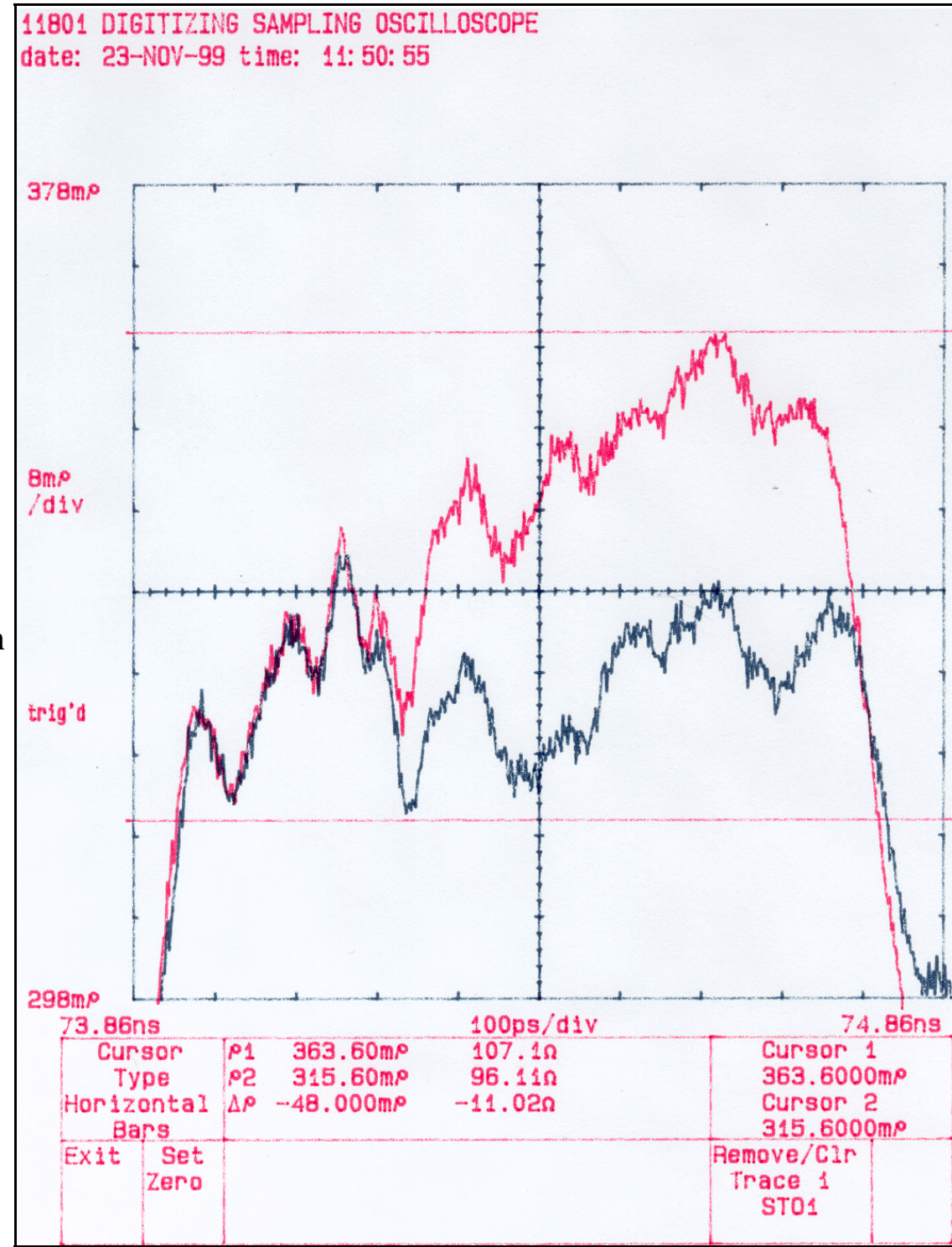

GHz antenna. The overall

Figure 7. The TDR plot of the $0.5-1 \mathrm{GHz}$ antenna. The black plot is dimension of the $1-2 \mathrm{GHz}$ the antenna in the mockup of the beampipe. The red curve is the antenna with a $10 \mathrm{~mm}$ bigger aperture. A mismatch is introduced when design was multiplied by a the aperture is changed.

factor of two and the design iteration began. The hardest part of the design was to create a successful launch from the microstrip mode to the coupler mode. A picture of the $0.5-1 \mathrm{GHz}$ antenna is shown in Fig. 6. This procedure was iterated upon several times using a razor blade and copper tape. The resulting TDR measurement is shown in Fig. 7. 
The black curve in Fig. 7 is the final TDR measurement for the 0.5-1 GHz antenna. The impedance of the antenna was designed to be $100 \Omega$ and the final measurement (or black curve in Fig. 7) shows that it is $100+1.5 /-3.2 \Omega$. The black curve was measured with the assembly of Fig. 5 closed tightly.

The red curve of Fig. 7 shows the TDR measurement with the assembly lifted $10 \mathrm{~mm}$, or equivalently, a $10 \mathrm{~mm}$ larger beam aperture. The impedance raises from $102 \Omega$ maximum to $107 \Omega$

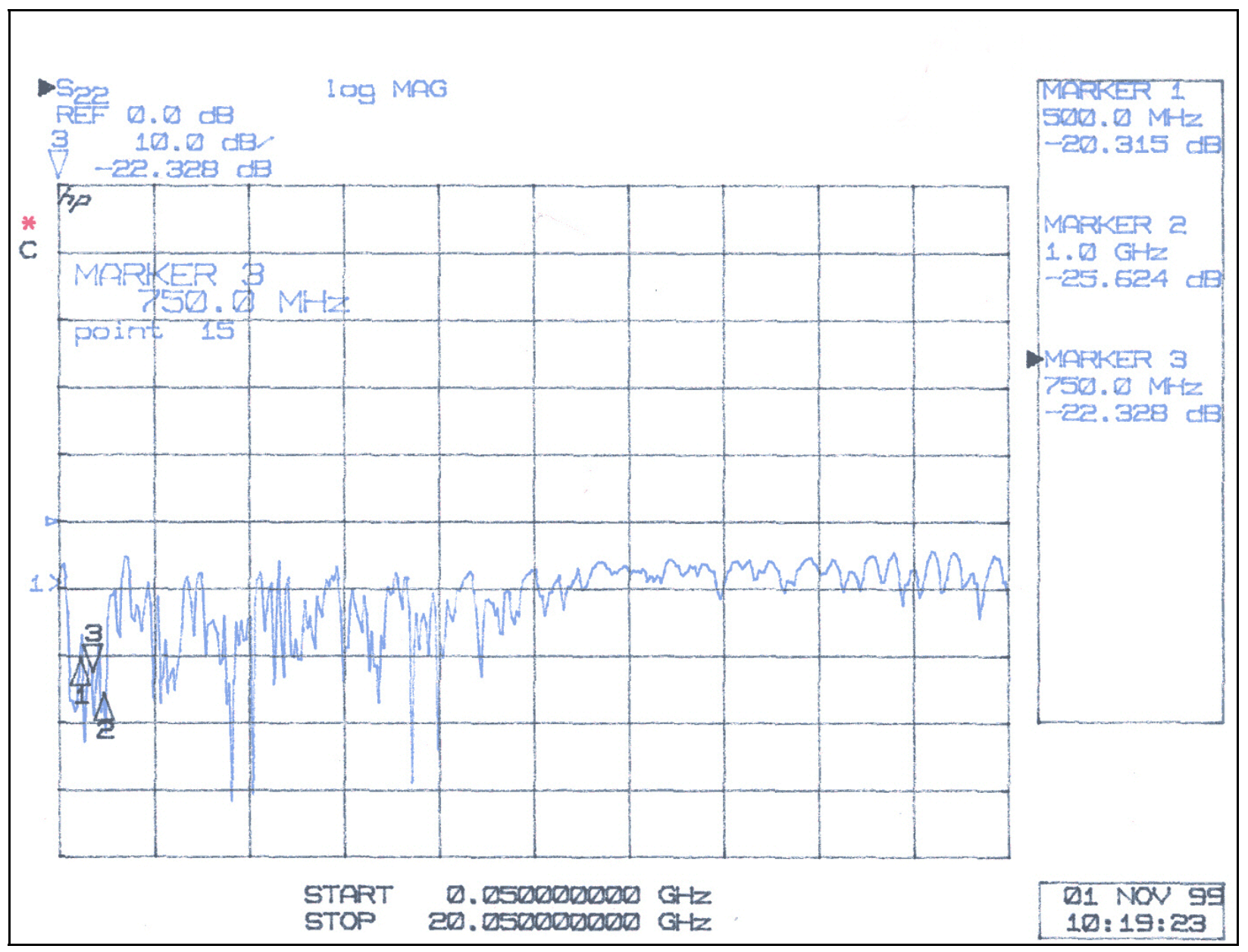

Figure 8. The reflection measurement of the $0.5-1 \mathrm{GHz}$ antenna in the closed assembly in Fig. 5. The matching network was tuned such that the $0.5-1 \mathrm{GHz}$ band is matched properly. The measurement shows that the antenna is well matched $\left(\mathrm{s}_{11}<-15 \mathrm{~dB}\right)$ in band. 
maximum. It is desired that the antenna have as constant of impedance as possible for it to have the best microwave match. Having a poor microwave match affects both the pickup as well as kicker behavior of the antenna. This situation will exist in the Recycler from reusing the antennas that were designed for the aperture in the Antiproton Source. It is important to note that the launch is similar between the open and closed aperture. This geometry of the microwave circuit in the immediate vicinity of the launch is critical for the launch to occur successfully. The 1-2 GHz and 2-4 GHz system will therefore be increasingly less vulnerable to these effects.

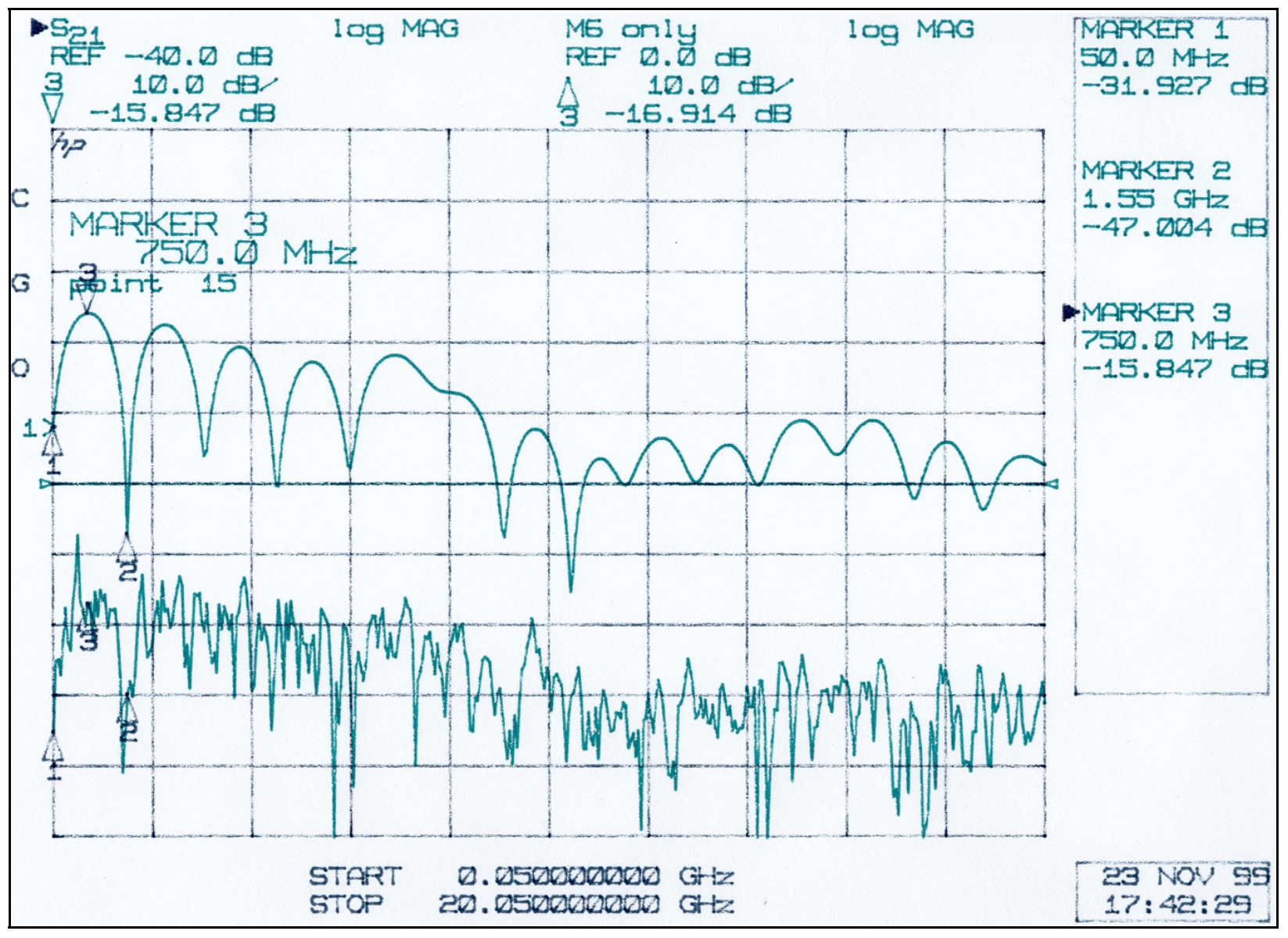

Figure 9. The SWM of the $0.5-1 \mathrm{GHz}$ antenna. The bottom measurement is the actual measurement. The top measurement utilized gating to smooth the measurement and to more accurately determine the behavior of the antenna and to de-embed the behavior of the wire reflections and matching networks. 
The antenna was then measured with a HP 8510 network analyzer. The network analyzer was calibrated using a full two port calibration over a broad band in a stepped frequency mode. The broad band calibration was performed so that the antenna could be examined in the time domain. The results of the reflection measurement are shown in Fig. 8. The antenna was matched using a Chebyshev matching transformer/network and was designed to have a good match from $450 \mathrm{MHz}$ to $1.1 \mathrm{GHz}$. The reflection measurement shows that the antenna is well matched over the $0.5-1 \mathrm{GHz}$ bandwidth. Next the antenna was measured using a SWM. The results of this measurement are shown in

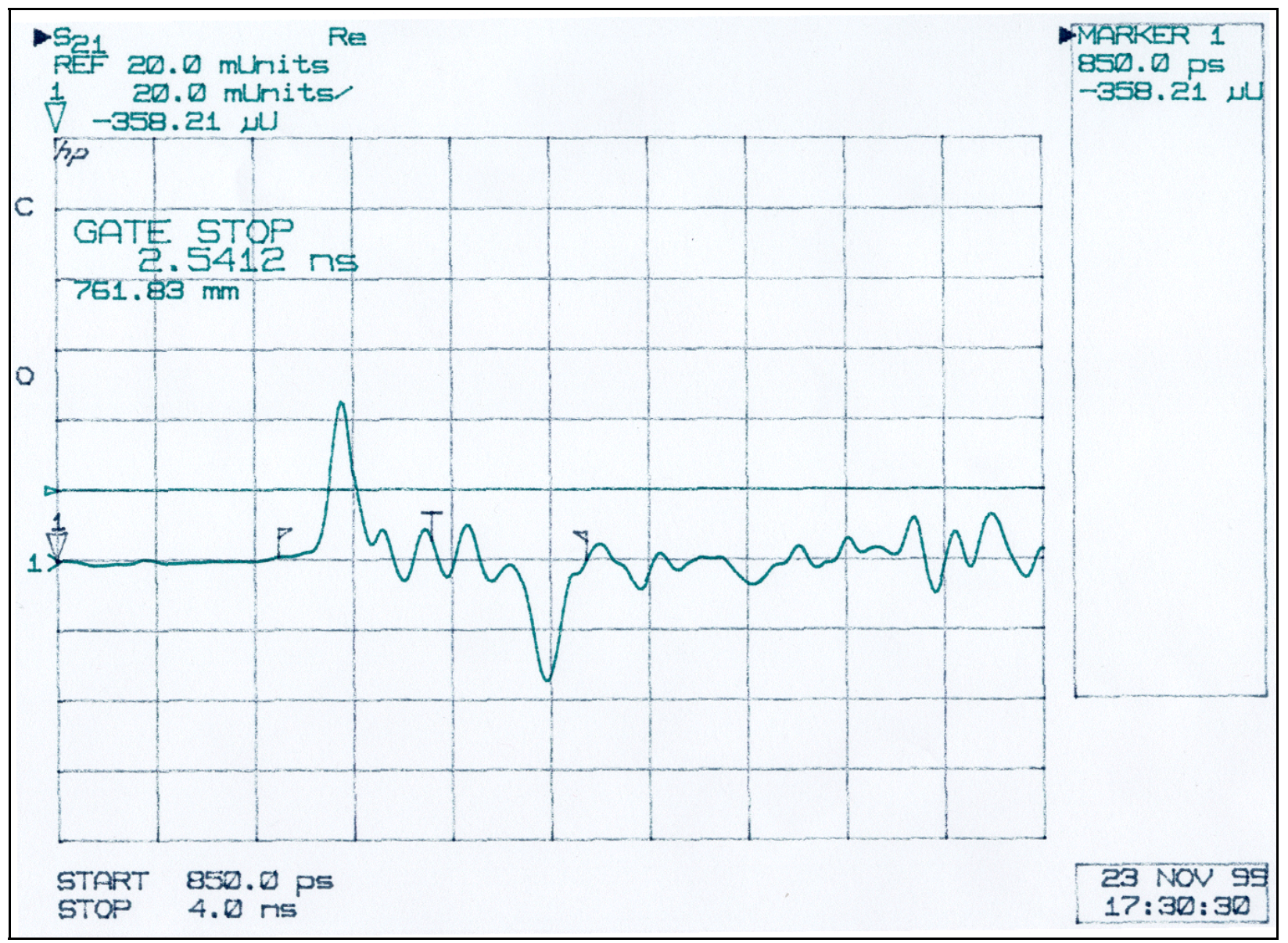

Figure 10. The time domain data of the actual measurement, or data on the bottom, in Fig. 9. The impulses of Eq. 1 are the two big peaks. This measurement will be gated at the positions between the flags and is shown in Fig. 11. 
Fig. 9. The bottom curve is the actual measurement depicting the transfer of energy from the wire through the antenna. This measurement shows reflections caused by the wire and matching networks.

The measurement was cleaned up by using the time domain option on the network analyzer. The gating operation was performed as follows. First the frequency domain data was collected. The data was then looked at in the time domain assuming an impulse response. The impulse response data is shown in Fig. 10. Because a limited bandwidth was used (only $20.05 \mathrm{GHz}$ ), the accuracy of the time

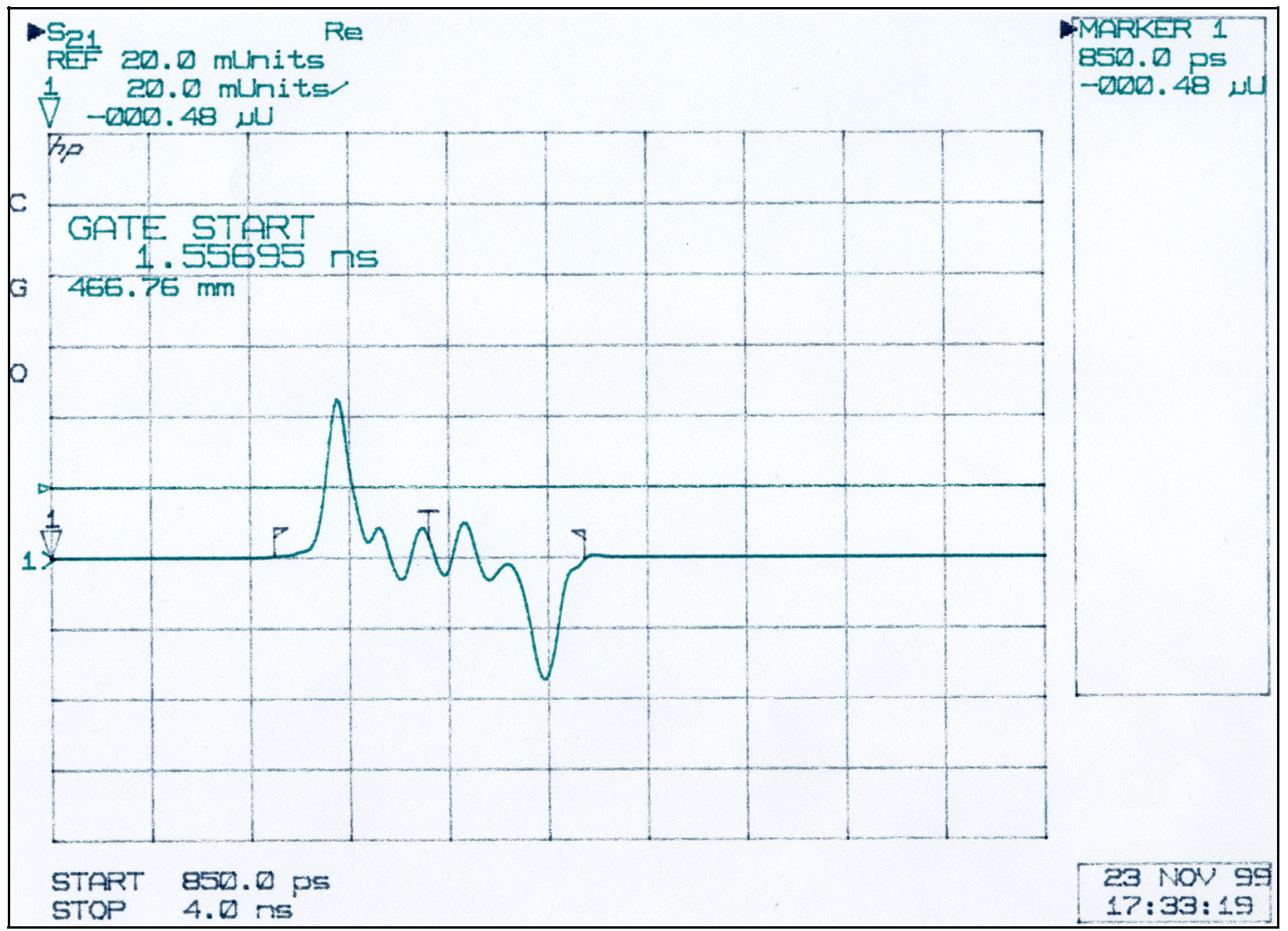

Figure 11. A plot of the gated data of Fig. 10. The gated data is then examined back in the frequency domain. The reflections caused by the wire are removed and a more accurate picture of how the pickup/kicker behaves can be examined. The small curves between the two peaks is caused by reflections in the matching network as well as in the antenna itself. The gated frequency data is shown in the top curve in Fig. 9. 
domain data is somewhat limited and the impulse functions described in Eq. 1 are not necessarily impulses but have a definite time spread. The data was gated between the flags and the resulting waveform is shown in Fig. 11. Figure 11 shows that the gating makes all signals outside of the gate to be zero. The gated data can be then examined back in the frequency domain. This is the data depicted at the top of the measurement of Fig. 9. This measurement shows the frequency repetitive behavior of the pickup/kicker. It also shows that the center frequency is $750 \mathrm{MHz}$ and the coupling to the beam is $-15.8 \mathrm{~dB}$.

\section{Conclusion}

The design of the $0.5-1 \mathrm{GHz}$ pickup is an experimental process and requires several iterations for a robust design. Extensive use of TDR's and NWA's ensure that the progress of the design goes in the correct direction. The antenna is tuned with both copper tape and with razor blades. The SWM is an excellent bench technique to determine the behavior of the antenna. The final determination of the center frequency of the antenna should be determined with the beam itself. Use of gating in the SWM technique can smoothen the data and help provide an excellent diagnostic tool for realizing the final form of the antenna.

Acknowledgments: I would like to thank John Marriner, Dave McGinnis, Paul Derwent, and Dave Vander Muelen for our insightful conversations and help in beginning this project. I also want to thank Christine Ader for her help in designing and fabricating the mockup assembly of the Recycler beampipe and to Don Poll and Gary Golinski for their help in the antenna fabrication and layout. 
